\title{
The possible protective effect of L-arginine against 5-fluorouracil-induced nephrotoxicity in male albino rats
}

\author{
M.H. Badawoud ${ }^{1}$, E.B. Elshal' ${ }^{2}$ A.I. Zaki1, 3, H.A. Amin ${ }^{1,4}$ \\ ${ }^{1}$ Department of Anatomy, Faculty of Medicine, King Abdulaziz University, Jeddah, Saudi Arabia \\ ${ }^{2}$ Department of Anatomy, Faculty of Medicine for Girls, Al-Azhar University, Cairo, Egypt \\ ${ }^{3}$ Department of Anatomy, Faculty of Medicine, Alexandria University, Egypt \\ ${ }^{4}$ Department of Histology, Faculty of Medicine, Cairo University, Egypt
}

[Received: 17 February 2017; Accepted: 3 April 2017]

\begin{abstract}
5-fluorouracil (5-FU) is a potent antineoplastic agent used for the treatment of various malignancies. The L-arginine nitric oxide (NO) pathway involved in the pathogenesis of chemotherapy induced kidney damage. This work investigated the beneficial mechanism of L-arginine supplementation in 5-FU induced nephropathy. Eighty male Wistar rats were divided into four equal groups: control group; L-arginine group (378 mg/rat/day for 4 weeks); 5-FU group (189 mg/rat/week for 4 weeks) and L-arginine for 1 week before and 4 weeks concomitant with 5-FU group. At the end of experiment, the kidney functions were assessed and kidneys specimens were processed for paraffin sections and stained with haematoxylin and eosin (H\&E), Masson's trichome (MT) and periodic acid-Schiff (PAS) stains. Other sections were processed for immunohistochemical demonstration of caspase-3 and inducible NO synthase (iNOS). Image analyser was used to analyse the results morphometrically and statistically. L-arginine administration to 5-FU treated animals elicited significant reduction in serum urea and creatinine levels, urine volume, urinary protein excretion and kidney/body weight ratio in comparison to fluorouracil treated group. L-arginine improved glomeruloscelerosis, degeneration of convoluted tubules and interstitial fibrosis in 5-FU treated animals. L-arginine attenuated effectively some biochemical and histological changes in 5-FU nephrotoxicity. (Folia Morphol 2017; 76, 4: 608-619)
\end{abstract}

Key words: L-arginine, nephrotoxicity, 5-fluorouracil

\section{INTRODUCTION}

Anticancer drugs under ideal circumstances would eliminate cancer cells without damaging normal tissues. However, no agents now available are completely devoid of toxicity [11]. 5-fluorouracil (5-FU) is regarded as the widespread agent in the management of colorectal cancer [13] and it's got activity against many solid tumours, including cancers in the breast, stomach, pan- creas, oesophagus, liver, head and neck, and anus [11]. Although, 5-FU generated acceptable outcome, it was considered a nephrotoxic compound [25]. Moreover, it is a pyrimidine fluorinated analogue and classified as being an antimetabolic agent that inhibits the synthesis of both DNA and RNA in normal and tumour cells [42].

L-arginine is really a semi essential amino acid and is thought to be a principle source for nitric oxide 
(NO) generation through NO synthase (NOS) [29]. This temporary molecule (NO) may play a crucial role in regulating kidney function in normal and pathological conditions [14]. Three NOS isoforms have been expressed in the kidney [16]. Endothelial NOS (eNOS) has a role in regulation of renal blood flow. Neuronal NOS (nNOS) is found mainly in the macula densa and has a role in secretion of renin. Inducible NOS (iNOS) is found in the kidney in pathologically affected mesangium and tubules [33]. The physiological importance of iNOS in kidney tubules is unclear [15].

In many studies exogenous L-arginine may protect renal tissue against toxic or ischaemic injury [39]. L-arginine/NO pathway defects are already suggested to have a main role in the pathogenesis of kidney affection. The renoprotective effect of L-arginine was postulated due to increased total renal blood flow and NO content [28]. Although many experimental studies have been performed on the renoprotective effect of L-arginine with conflicting results, there are limited studies on histological and immunohistochemical basis of this effect.

Based on all previous observations, the present study investigated on biochemical, histological and immunohistochemical basis the possible beneficial influence of L-arginine on nephrotoxicity caused by 5-FU administration in rats.

\section{MATERIALS AND METHODS}

\section{Ethical approval}

The procedures were conducted in accordance with guidelines and protocols reviewed and approved by the ethical committee for animal care and use in King Fahd Medical Research Centre (KFMRC), KAU, Jeddah, Saudi Arabia, which are in accordance with the guidelines of the Canadian Council on Animal Care.

\section{Drugs}

- 5-fluorouracil (Biosynth Company): was supplied in the form of ampoules of $250 \mathrm{mg}$ and intraperitoneally injected at $189 \mathrm{mg} / \mathrm{rat} /$ week $[18,34]$.

- L-arginine was purchased from Sigma-Aldrich Chemicals Co. (St. Louis, Missouri, USA). L-arginine solution was prepared by dissolving $40 \mathrm{~g}$ of L-arginine in $100 \mathrm{~mL}$ of normal saline to obtain concentration of $378 \mathrm{mg} / 0.9 \mathrm{~mL}$ and given orally. The calculated dose of L-arginine was based on preliminary data $[5,31]$ on its nephroprotective effect.

\section{Animals}

Eighty adult male Wister albino rats $(190-210 \mathrm{~g})$ were chosen for the study. They were maintained under normal laboratory conditions, and were received free access of normal laboratory chow and water ad libitum. Rats were acclimatised for a week before beginning the experiment.

\section{Experimental protocol}

Rats were randomly divided into four groups (20 rats each):

- Group I (control group): received intraperitoneal injection (IP) of normal saline solution $(3.78 \mathrm{~mL} /$ /week) for 4 weeks [18, 34];

- Group II (L-arginine group): received oral L-arginine $(378 \mathrm{mg} / \mathrm{rat} /$ day) for 4 weeks [5, 31];

- Group III (5-FU group): received IP of 5-FU (189 mg/rat/week) for 4 weeks [18, 34];

- Group IV (L-arginine group + 5-FU group): was treated with L-arginine ( $378 \mathrm{mg} / \mathrm{rat} /$ day) starting 1 week before 5 -FU which was given at the same dose, route and period as group III.

By the end of treatment, blood samples were collected from the tail vein of experimental groups and the serum was separated from each sample for assessing total proteins, albumin, urea and creatinine levels. Also the urine volume $(\mathrm{mL} / 24 \mathrm{~h})$ and urinary protein excretion $(\mathrm{mg} / 24 \mathrm{~h})$ were estimated.

\section{Absolute and relative kidney weight}

The mean weight of the rats was recorded; then the animals were sacrificed by decapitation. The animals were dissected and their kidneys were separated and weighed. Absolute and relative organ weights were determined.

\section{Histological and immunohistochemical techniques}

The right kidneys were separated and immediately fixed in $10 \%$ buffered formalin and processed for preparing histological sections $5 \mu \mathrm{m}$ thick. They were stained with haematoxylin and eosin (H\&E) and Masson's trichrome (MT) stains and periodic acid-Schiff's reaction (PAS) [8].

Other sections were immunohistochemically stained by avidin-biotin peroxidase method for detection of caspase-3 and iNOS expression [7]. Briefly, sections were deparaffinised, hydrated and then incubated overnight with the mouse monoclonal primary antibody to caspase-3 (Ab-7, Mouse Mab. MS.) at a dilution of 1:500 or rabbit polyclonal pri- 
Table 1. Body weight, absolute and relative kidney weight (KW/BW) of fluorouracil-treatment with or without L-arginine

\begin{tabular}{lccc}
\hline & BW [g] & KW [g] & KW/BW \\
\hline Group I & $295.5 \pm 3.4$ & $1.18 \pm 0.10$ & $0.40 \pm 0.02$ \\
Group II & $303.0 \pm 6.1$ & $1.15 \pm 0.08$ & $0.38 \pm 0.04$ \\
Group III & $218.5 \pm 5.3^{*} \dagger$ & $1.67 \pm 0.09^{*} \dagger$ & $0.76 \pm 0.05^{*} \dagger$ \\
Group VI & $283.9 \pm 5.4 \#$ & $1.26 \pm 0.09 \#$ & $0.44 \pm 0.03 \#$ \\
\hline
\end{tabular}

Data are represented as mean \pm standard deviation $(n=6) ; p<0.05$ was considered significant

${ }^{*}$ A significant change in comparison with control (group I)

tA significant change in comparison with $L$ arginine group (group II)

\#A significant change in comparison with fluorouracil group (group III)

mary antibody specific for iNOS enzyme (SC-650, Santa Cruz Biotechnology) at a dilution of 1:1000. Using a universal detection kits (Dakocytomation), biotinylated secondary antibodies form a complex with peroxidase conjugated streptavidin molecules. Sections were rinsed in phosphate buffered saline and few drops of biotinylated secondary antibodies were applied for $15 \mathrm{~min}$. Then, sections were rinsed and treated with the prepared diaminobenzidine tetra-hydro chloride (DAB) substrate chromogen solution for 15 min until the desired brown colour obtained. Finally, sections were counterstained with Mayer's haematoxylin.

\section{Morphometric studies}

Morphometric measurements were done using Image-Pro Premier system. Glomerular sclerosis was assessed as incremental degrees of PAS positive materials, obliteration of capillary lumina and the presence of amorphous hyaline material and scored based on the criteria of Romero et al. [35]. A minimum 40 glomeruli were randomly examined in every specimen with a magnification of $\times 400$ using Image-Pro Premier image analysis software. Sclerosis involving greater than $80 \%$ in the glomerular tuft was considered global, and sclerosis involving lower than $80 \%$ was considered segmental. Data are expressed as the percentage of glomeruli showing segmental or global sclerosis.

The average percentages of scored histopathological lesions of renal tissues such as tubular damage and atrophy, tubulo-interstitial infiltration and interstitial fibrosis were recorded according to the criteria of Romero et al. [35]. The degree of injury includes five scores depending on the ratio of affected area to total area of the section in the field of vision: 0 - affected area $<10 \%, 1-10 \%<$ affected area $<20 \%, 2-20 \%>$ affected area $<40 \%, 3-40 \%$
$>$ affected area $<60 \%, 4-60 \%<$ affected area $<80 \%, 5-$ affected area $>80 \%$. The mean score from 10-15 sections of each specimen were recorded.

The area per cent of caspase-3 and iNOS immuno-stained slides was studied and compared among the different groups used in this study. The area per cent of the immunostaining was measured in 10 microscopic fields (original magnification, $\times 200$ ) for every animal and the mean values were calculated.

\section{Statistical analysis}

Comparison between different groups was statistically done using one way analysis of variance (ANOVA) and then by multiple comparison test to evaluate the main difference between various groups. Differences were considered statistically significant when $\mathrm{p}<0.05$.

\section{RESULTS}

Effect on BW, KW and relative $\mathrm{KW}$ to $\mathrm{BW}$ (Table 1)

Fluorouracil treated rats (group III) had significantly lower body weight (BW) than controls. However, fluorouracil treated rats that additionally received L-arginine (group IV) were significantly heavier than the rats receiving fluorouracil alone. There was no significant difference between L-arginine (group II) and control groups.

Moreover, a significant increase in kidney weight $(\mathrm{KW})$ was shown in fluorouracil treated group. Animals co-treated with both L-arginine and fluorouracil showed a significant reduction in $\mathrm{KW}$ in comparison with fluorouracil treated group (group III).

There was a significant increase in $\mathrm{KW}$ to $\mathrm{BW}$ $(\mathrm{KW} / \mathrm{BW})$ ratio in the fluorouracil group $(\mathrm{p}<0.05)$. L-arginine treatment in group IV decreased KW/BW ratio compared to fluorouracil group as shown in Table 1. 
Table 2. Renal function markers of rats treated with fluorouracil with or without L-arginine

\begin{tabular}{lcccc}
\hline & Albumin [g/dL] & Total proteins [g/dL] & Creatinine [mg/dL) & Urea [mg/dL] \\
\hline Group I & $3.42 \pm 0.13$ & $6.0 \pm 0.3$ & $0.62 \pm 0.20$ & $33.62 \pm 0.13$ \\
Group II & $3.47 \pm 0.17$ & $7.0 \pm 0.5$ & $0.65 \pm 0.07$ & $33.57 \pm 0.17$ \\
Group III & $2.13 \pm 0.18^{*} \dagger$ & $4.1 \pm 1.5^{*} \dagger$ & $1.10 \pm 0.12^{*} \dagger$ & $85.13 \pm 0.18^{*} \dagger$ \\
Group VI & $3.35 \pm 0.04^{\#}$ & $7.3 \pm 1.1^{\#}$ & $0.75 \pm 0.06^{\#}$ & $35.33 \pm 0.04^{\#}$ \\
\hline
\end{tabular}

Data are represented as mean \pm standard deviation $(n=6) ; p<0.05$ was considered significant

${ }^{*}$ A significant change in comparison with control (group I)

†A significant change in comparison with L-arginine group (group II)

\#A significant change in comparison with fluorouracil group (group III)

Table 3. Urine volume and urinary proteins excretion in rats treated with fluorouracil with or without L-arginine

\begin{tabular}{lcc}
\hline & $\begin{array}{c}\text { Urine volume } \\
{[\mathrm{mL} / 24 \mathrm{~h}]}\end{array}$ & $\begin{array}{c}\text { Urinary proteins } \\
{[\mathrm{mg} / 24 \mathrm{~h}]}\end{array}$ \\
\hline Group I & $11.4 \pm 0.9$ & $6.0 \pm 0.4$ \\
Group II & $12.3 \pm 0.8$ & $7.0 \pm 0.7$ \\
Group III & $29.0 \pm 2.3^{*} \dagger$ & $24.1 \pm 1.5^{*} \dagger$ \\
Group VI & $15.3 \pm 3.4^{\#}$ & $5.8 \pm 1.1^{\#}$ \\
\hline
\end{tabular}

Data are represented as mean \pm standard deviation $(n=6)$;

$p<0.05$ was considered significant

${ }^{*}$ A significant change in comparison with control (group I)

†A significant change in comparison with L-arginine group (group II)

\#A significant change in comparison with fluorouracil group (group III)

\section{Biochemical changes}

Effect on serum total proteins, albumin, urea and creatinine levels. Table 2 illustrates serum total proteins, albumin, urea and creatinine levels in rats receiving fluorouracil treatment with or without L-arginine. Fluorouracil-treated rats exhibited a considerable increase in serum urea and creatinine levels compared with apparent depletion of serum total protein and albumin contents. However, fluorouracil-treated group protected by L-arginine supplementation showed marked amelioration but was still below the normal value.

Effect on urine volume $(\mathrm{mL} / 24 \mathrm{~h})$ and urinary protein excretion ( $\mathrm{mg} / \mathbf{2 4} \mathrm{h}$ ) (Table 3 ). In experimental group treated with fluorouracil, there was a detected increase in $24 \mathrm{~h}$ urine volume and total urinary protein in comparison with the control group. However, L-arginine treatment of fluorouracil-intoxicated rats led to marked amelioration but was still not matched with the control animals.

N.B. No significant difference was detected between control untreated and L-arginine-treated controls at all parameters.

\section{HISTOLOGICAL RESULTS}

Group I (control group). The control kidney had normal histological architecture, Examination of $H \& E$ stained sections of kidneys of this group showed that the renal corpuscle was formed of a glomerular tuft of blood capillaries surrounded by the Bowman's capsule which had a parietal layer lined with simple squamous epithelium and inner concave visceral layer lined by round podocyte cells with deeply stained nuclei. In between the two layers there was a capsular space or (urine space). The proximal convoluted tubules had narrow lumen occupied by striated brush borders and a regular basal lamina lined by a single layer of pyramidal cells with eosinophilic cytoplasm and central rounded nuclei. Distal convoluted tubules lined by a relatively large number of cuboidal epithelial cells. The lumens of the distal tubules were wider than the proximal tubules and their cytoplasm was less acidophilic and the nuclei were rounded (Fig. 1A).

Collagen fibres were found to be of minimal amounts and were confined to the Bowman's capsule, around the tubules and basal laminae of glomerular capillaries (Fig. 1B).

PAS positive materials were seen in the basement membrane of renal tubules in addition to the brush border of the proximal convoluted tubules. Intraglomerular PAS positive material was also detected (Fig. 1C).

Group II (L-arginine group). The renal architecture in L-arginine treated animals exhibited normal histological architecture with no changes detected in this group as compared to group I (Fig. 2A). Collagen fibre distribution and PAS positive areas were also similar to control sections (Fig. 2B, C).

Group III (fluorouracil group). In fluorouracil treated rats, areas of glomerular and tubular degen- 

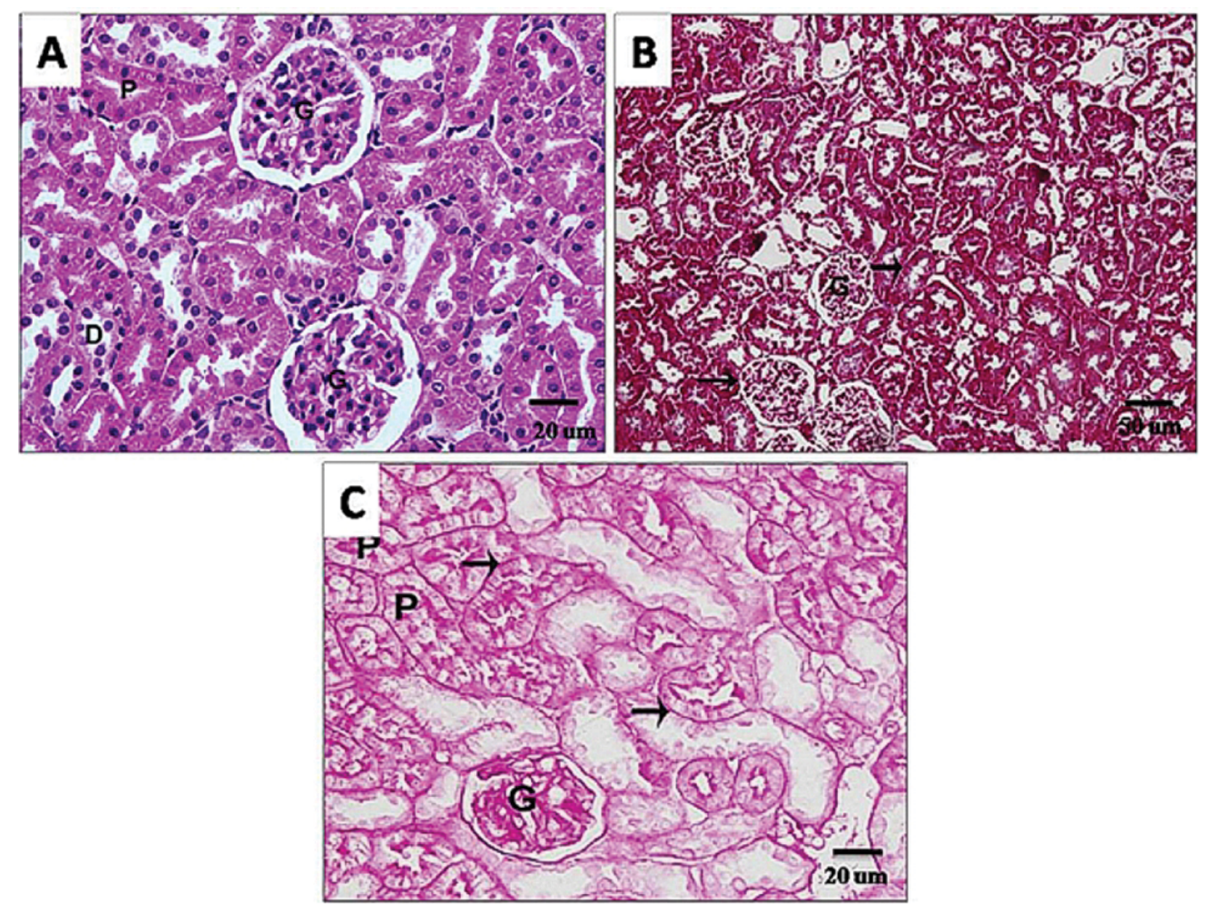

Figure 1. Photomicrographs of sections in the kidney of a control rat (group I) showing: (A) Normal renal architecture: glomerulus (G), proximal (P) and distal (D) convoluted tubules haematoxylin and eosin (H\&E), scale bar $=20 \mu \mathrm{m}$; (B) Normal distribution of collagen fibres in the glomerulus (G), Bowman's capsule and surrounding the tubules (arrows) Masson's trichrome, scale bar $=50 \mu \mathrm{m}$; (C) strong periodic acid-Schiff (PAS) reaction in the glomeruli (G), brush border of the proximal convoluted tubules (P), basal lamina (arrows) of tubules PAS, scale bar $=20 \mu \mathrm{m}$.
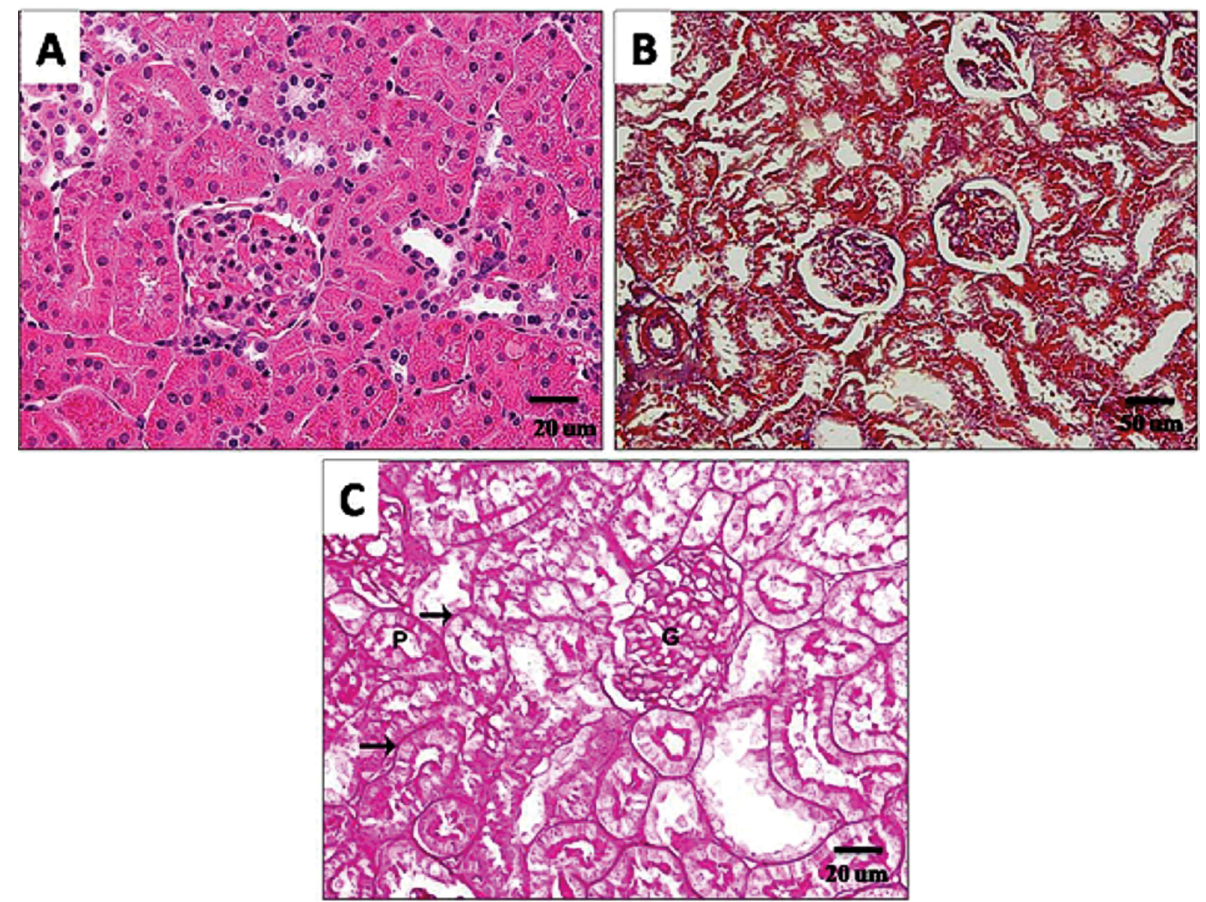

Figure 2. Photomicrographs of sections in the kidney of L-arginine treated rat (group II) showing: (A) Normal pattern of renal architecture haematoxylin and eosin (H\&E), scale bar $=20 \mu \mathrm{m}$; (B) Normal distribution of collagen fibres in the glomeruli, and surrounding the tubules Masson's trichrome, scale bar $=50 \mu \mathrm{m}$; (C) Strong periodic acid-Schiff (PAS) reaction in the glomeruli (G), brush border of the proximal convoluted tubules (P) and basal laminae of tubules (arrow) PAS, scale bar $=20 \mu \mathrm{m}$. 

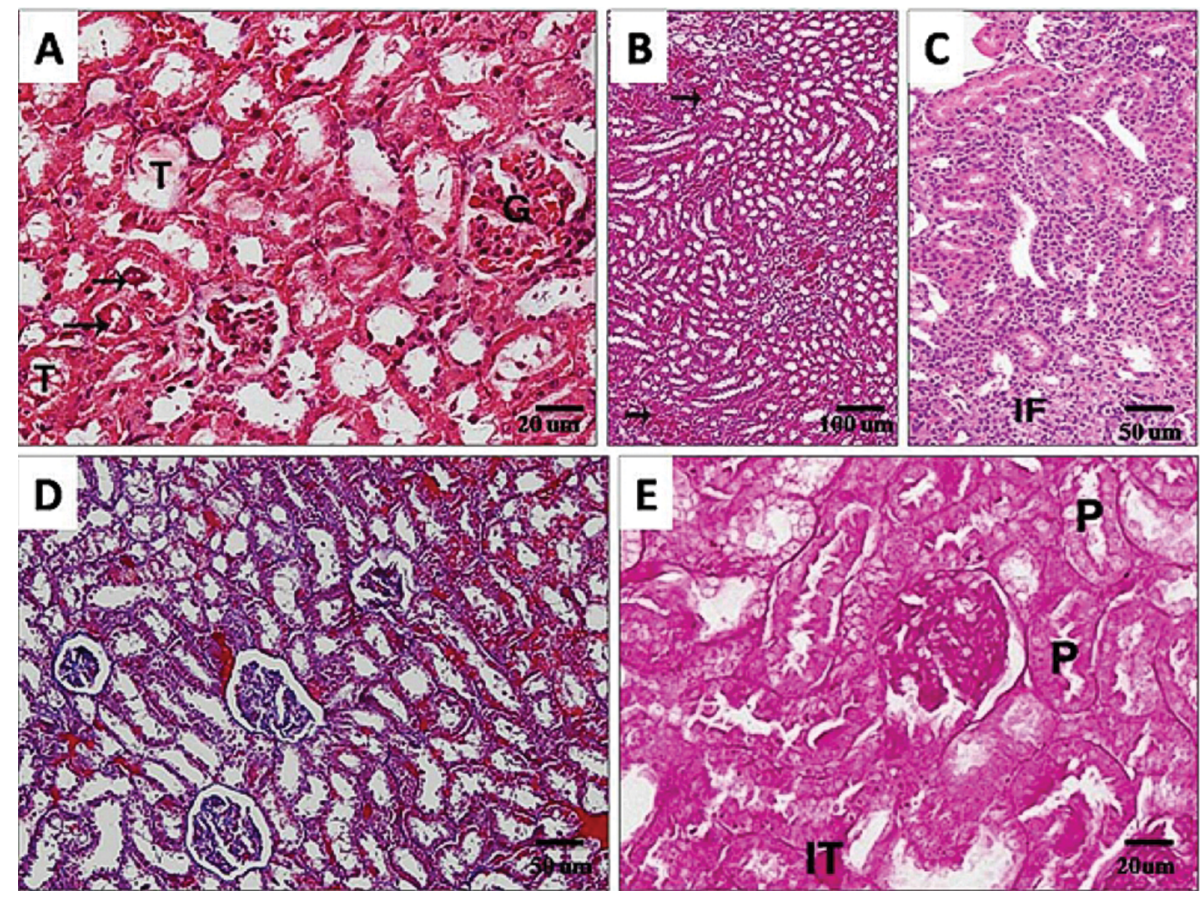

Figure 3. Photomicrographs of sections in the kidney of a fluorouracil treated rat (group III) demonstrating: (A) Dilated and congested glomerular capillary loops $(\mathrm{G})$ and vacuolisation of tubular epithelial cells $(\mathrm{T})$. Homogenous eosinophilic casts are seen in some tubules (arrows) haematoxylin and eosin (H\&E), scale bar $=20 \mu \mathrm{m}$; (B) Dilated and congested blood vessels and interstitial haemorrhages (arrows) $\mathrm{H} \& \mathrm{E}$, scale bar $=100 \mu \mathrm{m}$; (C) Extensive interstitial mononuclear infiltrating cells (IF) surrounding destructed tubules H\&E, scale bar $=50 \mu \mathrm{m}$; (D) Increased intraglomerular and peritubular collagen deposition Masson's trichrome, scale bar $=50 \mu \mathrm{m}$; (E) Increased intraglomerular periodic acid-Schiff (PAS) positive material. Most of proximal convoluted tubules shows weak reaction in their brush border (P) with focal interstitial strong reaction (IT) PAS, scale bar $=20 \mu \mathrm{m}$.

eration were seen among apparently normal ones. The epithelial lining of the affected tubules showed either pyknotic or vacuolated degeneration. Their tubular lumina appeared swollen with the presence of cellular debris. Hyaline casts were also observed within some tubular lumina (Fig. 3A). There were different patterns of glomerular damage such as glomerular atrophy, lobulation and glomerular sclerosis. Interstitial and periglomerular leukocytic infiltrations were detected (Fig. 3B, C). Dilated and congested glomerular capillaries were also seen (Fig. 3A). The renal cortex and medulla exhibited multiple congested capillaries, interstitial mononuclear cellular infiltration and extravasated blood cells (Fig. 3B, C).

Masson trichrome stained sections revealed increased intraglomerular and peritubular collagen fibres (Fig. 3D).

The glomerular mesangium was mostly expanded by increase of intraglomerular PAS positive material. Some tubules showed strong PAS reaction in their basal laminae while others revealed weak PAS reaction in their brush border. Few areas of interstitial tissue showed intense reaction (Fig. 3E).
Group IV (L-arginine + fluorouracil group). L-arginine supplementation to fluorouracil-treated group, showed improvement majority of renal corpuscles had narrow urinary space, regular glomerular cellularity. The proximal convoluted tubules cells showed vesicular rounded basal nuclei with acidophilic granular cytoplasm and characteristically narrow lumen. The distal convoluted tubules showed less acidophilic cytoplasm and characteristically wide lumen (Fig. 4A). Normal pattern of collagen fibres in the Bowman's capsule and around the tubules (Fig. 4B) and PAS positive staining structures were detected in the glomeruli in the basement membrane of the parietal layer of Bowman's capsules, in the basement membrane of renal tubules and in the brush border of the proximal convoluted tubules (Fig. 4C).

\section{Inducible NO and caspase-3 immunohistochemistry}

Inducible nitric oxide synthase was immunohistochemically detected in the cytoplasm of the tubular cells in control group. Higher expression was seen in fluorouracil treated group (group III) which was decreased by co-treatment of fluorouracil and L-arginine in renal tissues. No detectable difference was seen 


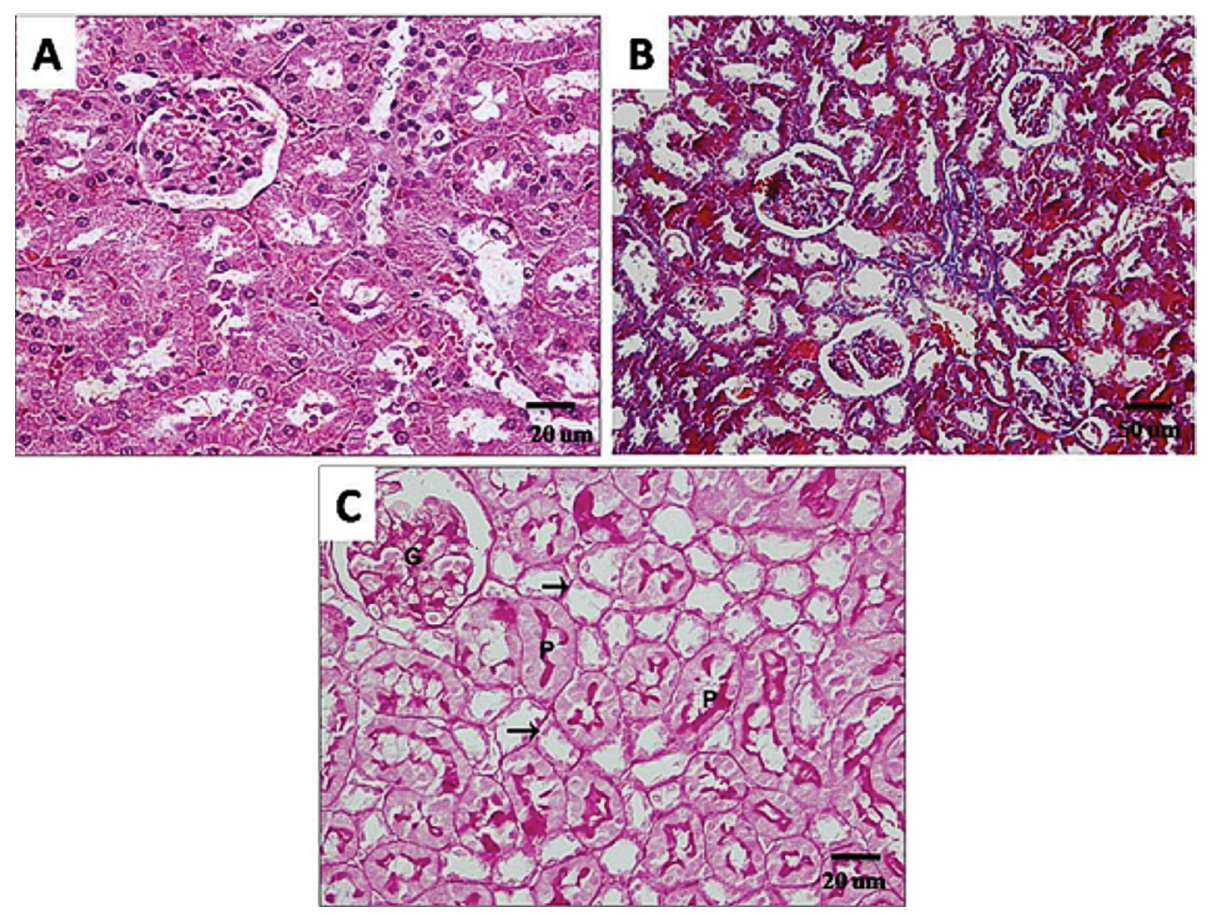

Figure 4. Photomicrographs of sections in the kidney of a rat co-treated with fluorouracil and L-arginine (group IV) showing: (A) Within normal renal architecture haematoxylin and eosin $(\mathrm{H} \& \mathrm{E})$, scale bar $=20 \mu \mathrm{m}$; (B) Within normal distribution of collagen fibres in the glomerulus, Bowman's capsule and surrounding the tubules Masson's trichrome, scale bar $=50 \mu \mathrm{m}$; (C) Strong periodic acid-Schiff (PAS) reaction in the glomeruli (G), brush border of the proximal convoluted tubules (P), basal laminae of tubules (arrows) PAS, scale bar $=20 \mu \mathrm{m}$.

between group II (L-arginine group) and control rats (Fig. 5A-D).

Caspase-3 immunohistochemistry showed nearly negative staining affinity in control and L-arginine-treatment. However, in experimental group treated with fluorouracil, there was an apparent increase in caspase-3 cytoplasmic expression, and these was markedly reduced in fluorouracil-treated group supplemented L-arginine (Fig. 6A-D).

\section{Quantitative morphometric results}

The quantitative morphometric histological results are summarised in Table 4. In group III (fluorouracil treated rats), $13.8 \%$ of glomerular tuft area was segmentally sclerosed and $2.0 \%$ was globally sclerosed. L-arginine treatment (group VI) decreased significantly the glomerulosclerosis compared to group III.

Tubular degeneration and atrophy, interstitial cell infiltration and interstitial fibrosis were demonstrated at a mean score of $4.2,1.7$ and 1.2 , respectively in group III (fluorouracil group). L-arginine (group IV) significantly decreased the mean score of these lesions. Meanwhile, no significant difference was detected between groups; II and IV and the control group.
The mean area per cent of iNOS immunostaining in control sections was $4.112 \pm 0.703$. It significantly increased in group III (fluorouracil group) to $18.549 \pm 1.34$. However, nonsignificant difference was found in groups II (L-arginine group) and IV (L-arginine + fluorouracil group) in comparison to control rats; $4.624 \pm 0.112$ and $5.391 \pm 0.230$, respectively. Moreover, the mean area per cent of caspase-3 immunoexpression indicated a highly significant increase in group III $(12.8 \pm 1.17)$ in comparison with control rats $(2.5 \pm 0.92)$ and a significant decrease in group IV $(7.3 \pm 0.51)$ in comparison with group III.

\section{DISCUSSION}

5-fluorouracil is a widely used chemotherapeutic drug, due to its efficacy in varieties of human malignancies; however, it has hepatotoxic and nephrotoxic side effects [21]. This organ toxicity is coupled with increased oxidative stress and apoptosis [34]. Therefore, the current work studied the possible protective effect of L-arginine treatment as a NO precursor against 5-FU induced nephrotoxicity.

In the present work, animals dissection showed that the decrease in body weight induced by 5 -FU might be 

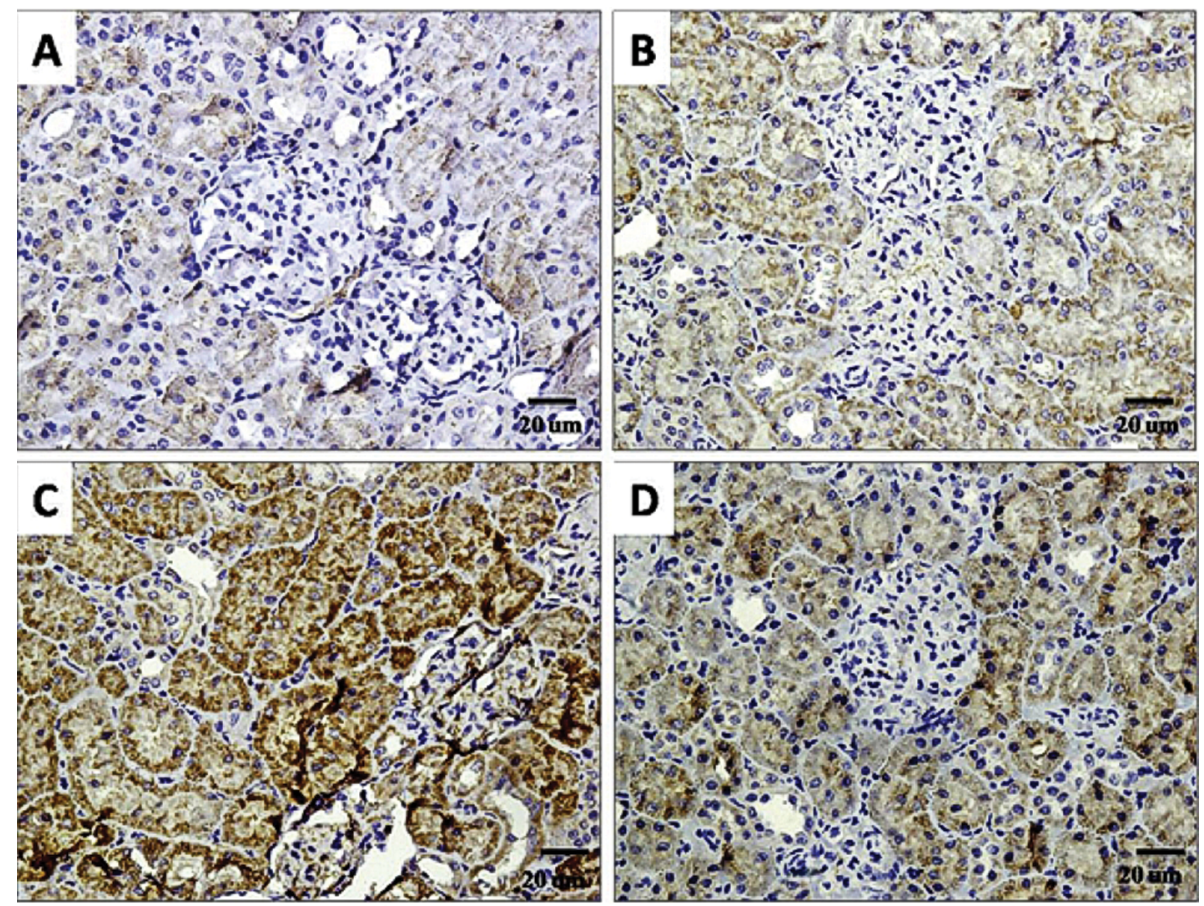

Figure 5. Photomicrographs of sections in the kidneys of a control rat (group I) showing very weak immunostaining of inducible nitric oxide synthase (iNOS) (A); B. L-arginine treated rat (group II) showing mild positive immunostaining; C. A fluorouracil treated rat (group III) showing increased area of strong positive iNOS immunostaining; D. A rat co-treated with fluorouracil and L-arginine (group IV) showing moderately iNOS positive immunostaining. iNOS, scale bar $=20 \mu \mathrm{m}$.
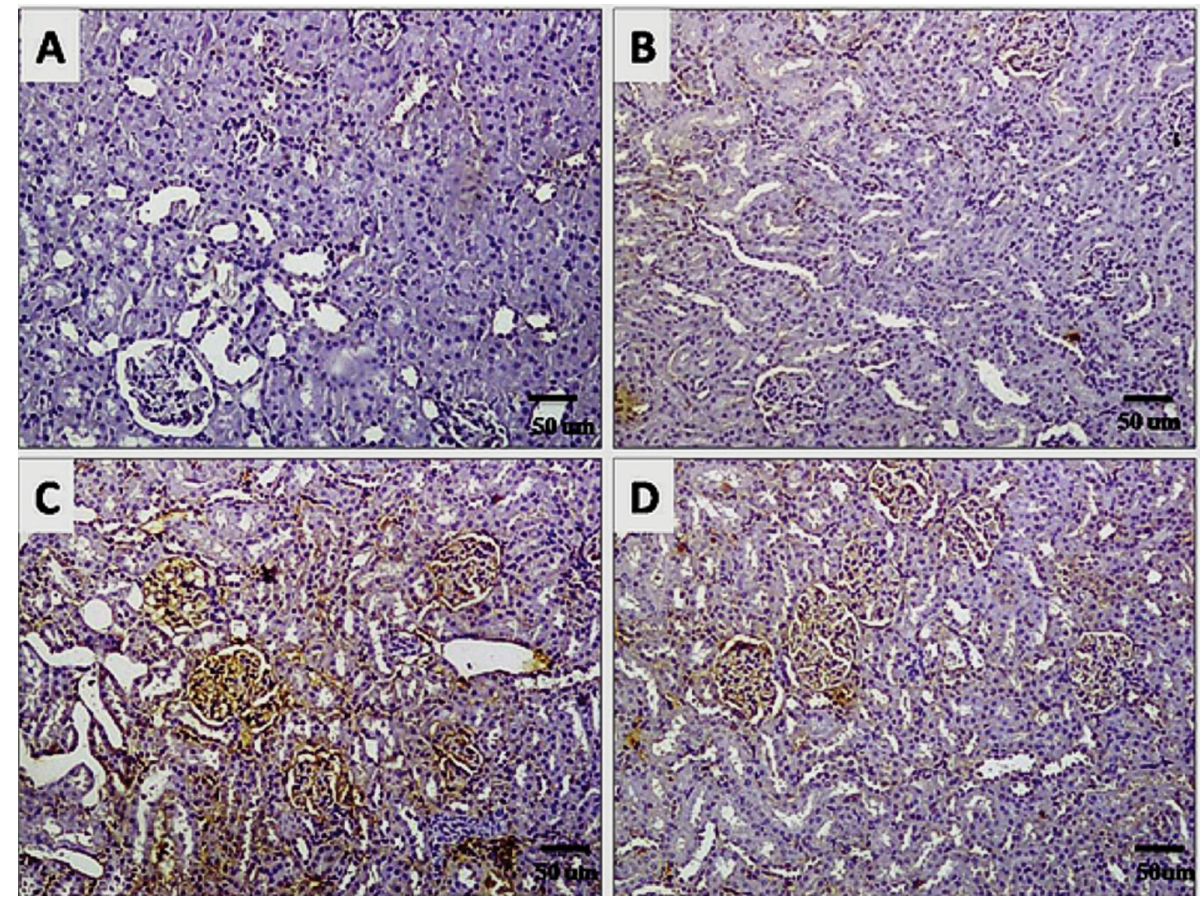

Figure 6. Photomicrographs of sections in the kidneys of a control rat (group I) showing nearly negative immunostaining of caspase-3 (A); B. L-arginine treated rat (group II) showing very weekly positive immunostaining; C. A fluorouracil treated rat (group III) showing darkly stained areas of positive immunoreactions; D. A rat co-treated with fluorouracil and L-arginine (group IV) showing lightly stained areas of immunostaining. caspase-3, scale bar $=50 \mu \mathrm{m}$. 
Table 4. Morphometry of histopathological lesions and immunohistochemical reactions

\begin{tabular}{|c|c|c|c|c|}
\hline & Group I & Group II & Group III & Group IV \\
\hline \multicolumn{5}{|l|}{ Glomerular sclerosis: } \\
\hline Normal (\%) & $98.1 \pm 0.5$ & $97.3 \pm 2.1$ & $84.2 \pm 4.1$ & $96.8 \pm 1.7$ \\
\hline Segmental (\%) & $1.9 \pm 0.13$ & $2.7 \pm 0.02$ & $13.8 \pm 3.2^{*} \dagger$ & $3.2 \pm 0.04^{\#}$ \\
\hline Global (\%) & 0.0 & 0.0 & $2.0 \pm 1.0^{*} t$ & 0.0 \\
\hline Tubular degeneration and atrophy $(0-5)$ & 0.0 & 0.0 & $4.2 \pm 0.10^{*} \dagger$ & 0.0 \\
\hline Interstitial infiltration (0-5) & 0.0 & 0.0 & $1.7 \pm 0.40^{*} \dagger$ & 0.0 \\
\hline Interstitial fibrosis (0-5) & 0.0 & 0.0 & $1.2 \pm 0.31^{*} \dagger$ & 0.0 \\
\hline The mean area per cent of iNOS & $4.11 \pm 0.7$ & $4.62 \pm 0.11$ & $18.55 \pm 1.34^{*} \dagger$ & $5.39 \pm 0.23^{\#}$ \\
\hline The mean area per cent of caspase-3 & $2.5 \pm 0.92$ & $3.8 \pm 0.02$ & $12.8 \pm 1.17^{*} \dagger$ & $7.3 \pm 0.51^{\#}$ \\
\hline
\end{tabular}

Data are represented as mean \pm standard deviation; $p<0.05$ was considered significant; iNOS - inducible nitric oxide synthase

${ }^{*} p<0.05$ in comparison with the control group (group I)

$t p<0.05$ in comparison with the $L$ arginine treated group (group II)

$\# \mathrm{p}<0.05$ in comparison with the fluorouracil treated group (group III)

due to loss of skeletal muscles and adipose tissue. This suggestion was also reported by Devlin [19]. In addition, a significant rise in relative $\mathrm{KW}$ to $\mathrm{BW}$ was in agreement with the results recorded by Saleh and El-Demerdash [37]. This increase in reno-somatic index might be due to the oedema of renal parenchyma caused by renal inflammation [2].

The biochemical findings in this work were in agreement with those obtained by El-Hoseany [20] and Rashid et al. [34] who reported that 5-FU administration led to impairment in kidney function as shown by increase in creatinine and urea and a significant reduction in total serum proteins and albumin. Nephrotoxicity induced by 5-FU was confirmed by histological changes including glomerular and tubular degeneration. Homogenous eosinophilic casts were seen in some tubules. Dilated and congested glomerular capillary loops were frequently observed. Obtained results are similar to those recorded previously by Ali and Al Moundhri [4] and Rashid et al. [34]. They confirmed that 5-FU and cisplatin severely impaired renal function.

Presently, treatment with L-arginine starting 1 week before 5-FU, apparently reduced its deleterious effects and protected the kidney from damage. This protection was clearly reflected by a significant decrease in kidney weights and in KW relative to $\mathrm{BW}$ and a rise in total serum proteins and albumin. Also, serum urea and creatinine returned nearly to its normal levels. These findings were also reported by Abo Zeid et al. [1]. The authors postulated that L-arginine treatment as a NO precursor caused a significant improvement of kidney functions in various forms of acute and chronic renal injury. The inductive effects of L-arginine might be due to a change in the level of endogenous NO. This effect was reported to protect against drug induced nephrotoxicity such as cyclosporine [31] and gentamycin [14] as well as in unilateral ureteral obstruction [26].

Nitric oxide is synthesized from L-arginine by three different isoforms of nitric oxide synthases (NOSs): neuronal (nNOS), inducible (iNOS) and endothelial nitric oxide synthase (eNOS). As NO has no specific receptors, its function and activity in the different pathophysiological conditions mainly depend on the site and concentration of its production and the surrounding mediators. It has a central function in neurotransmission, inflammatory processes and in the regulation of angiogenesis and vasodilatation [36].

Our immunohistochemical and morphometric results showed that iNOS was minimally expressed in normal renal tissue and highly expressed in damaged proximal tubule epithelial cells in 5-FU treated group. The present study attempted to clarify the consequence of modulation of iNOS on the extent of 5-FU induced nephrotoxicity by using L-arginine as a NO precursor.

Many studies showed that iNOS is low or not expressed in normal renal tissues, whereas several nephropathies were associated with substantial amounts of iNOS in the glomeruli and the renal interstitium [9, 12, 22, 24, 41]. Other studies, however, indicated that iNOS is expressed in large amounts in the normal renal tissue, localising mainly in the tubules, and that pathologic conditions, such as clinical and experimental chronic renal insufficiency, 
are associated with marked iNOS down regulation $[3,6,44]$. One possible reason for this disagreement is the wide heterogeneity of the experimental models studied so far. Additional discrepancy may arise from the fact that the primary antibodies used to detect iNOS come from several sources, because the behaviour of different antibodies directed against NOS isoforms can vary dramatically according to type (monoclonal vs. polyclonal), species in which the antibody was raised, and tissue in which the antibody is tested [17].

Schneider et al. [38] recorded that the histological changes induced by 5-FU might be related to the deprivation of internal L-arginine available for synthesis of NOS and subsequent uncoupling of constitutive NOS that triggers iNOS induction. The high expression of iNOS can be attributed to the compensatory increase in its level trying to increase the NO level to counteract the deleterious effect of 5-FU on the kidney. However, iNOS protein, once induced, produces large amounts of NO for a sustained period. This NO acts as a free radical and causes cytotoxicity in a variety of cells or tissues [15]. In the present work L-arginine improved the histological changes and consequently diminished the expression of iNOS compared to 5-FU group.

Schwartz et al. [40] reported that up-regulation of iNOS may lead to down-regulation of eNOS which is responsible for maintaining physiologic renal functions. The iNOS rapidly reacts with superoxide (O2-), resulting in the formation of the highly reactive oxidant peroxynitrite (ONOO-) rather than NO under conditions of absolute or relative L-arginine deficiency $[32,45]$. Oxidative stress linked to iNOS is mediated via lipid peroxidation and DNA damage [30]. Thus L-arginine treatment in the present work could protect against the tubular and glomerular histological changes by inhibiting iNOS activity and preventing the formation of ONOO- free radicals and consequently prevents the DNA damage induced by 5-FU.

5-fluorouracil caused strong caspase-3 immunostaining in the renal parenchyma. However, L-arginine treatment significantly attenuated apoptosis in both glomerular and tubular region by inhibiting caspase-3 activation. Therefore, L-arginine plays an important role in modulating oxidative stress and apoptosis induced by 5-FU. These results were concluded by Thant et al. [43] and Rashid et al. [34] who reported that apoptosis elicited by $5-\mathrm{FU}$ is a caspase-dependent process that includes activation of the initiator active caspase- 9 in addition to ef- fector caspase-3. Many researchers suggested that these changes are due to the free radical generation released in lipid peroxidation, cell membrane damage and apoptosis [46].

Several studies proposed a link between the antiapoptotic effect of L-arginine was secondary to the prevention of the formation of peroxynitrite anions in the renal tissue $[10,23,27]$.

\section{CONCLUSIONS}

In conclusion, administration of L-arginine was found to be powerfully protective in the model of nephropathy seen in fluorouracil treated rats. L-arginine has a tendency to preserve most of morphological, immunohistochemical and biochemical parameters towards normal values. Human trials are essential to prove this protective role.

\section{Acknowledgements}

This project was funded by the Deanship of Scientific Research (DRS), King Abdulaziz University, Jeddah, under grant no. (1433/140/386). The authors, therefore, acknowledge with thanks DSR technical and financial support. Principal investigator, Prof. Mohammed H. Badawoud.

\section{REFERENCES}

1. Abo Zeid, El Saka MH, Shafik NM. Effect of combination of $\mathrm{L}$-arginine and $\mathrm{N}$-acetyl cysteine in rat model of renal ischemia-reperfusion injury. J Am Sci. 2012; 8(10): 814-921, doi: 10.7537/j.issn.1545-1003.

2. Adejuwon A, Femi-Akinlosotu O, Omirinde JO, et al. Launaea taraxacifolia Ameliorates Cisplatin-Induced Hepato-renal Injury. Eur J Med Plants. 2014; 4(5): 528-541, doi: 10.9734/ejmp/2014/7314

3. Aiello $S$, Noris $M$, Todeschini $M$, et al. Renal and systemic nitric oxide synthesis in rats with renal mass reduction. Kidney Int. 1997; 52(1): 171-181, indexed in Pubmed: 9211360.

4. Ali BH, Al Moundhri MS. Agents ameliorating or augmenting the nephrotoxicity of cisplatin and other platinum compounds: a review of some recent research. Food Chem Toxicol. 2006; 44(8): 1173-1183, doi: 10.1016/j. fct.2006.01.013, indexed in Pubmed: 16530908

5. Amore A, Gianoglio B, Ghigo D, et al. A possible role for nitric oxide in modulating the functional cyclosporine toxicity by arginine. Kidney Int. 1995; 47(6): 1507-1514, indexed in Pubmed: 7543959.

6. Ashab I, Peer G, Blum M, et al. Oral administration of L-arginine and captopril in rats prevents chronic renal failure by nitric oxide production. Kidney Int. 1995; 47(6): 1515-1521, indexed in Pubmed: 7643519.

7. Bancroft JD, Cook HC. Immunohistochemical staining by standard avidin-biotin-peroxidase method. In: Bancroft JD, 
Cook HC. ed. Manual of histological techniques. Churchill, Livingstone 1984: 195-202.

8. Bancroft JD, Gamble M. Theory and practice of histological techniques. 5th ed. Churchill, Livingstone 2001: 173-175.

9. Bank N, Aynedjian HS, Qiu JH, et al. Renal nitric oxide synthases in transgenic sickle cell mice. Kidney Int. 1996; 50(1): 184-189, indexed in Pubmed:8807587.

10. Bidadkosh A, Derakhshanfar A, Rastegar A, et al. Antioxidant preserving effects of I-arginine at reducing the hemodynamic toxicity of gentamicin-induced rat nephrotoxicity: pathological and biochemical findings. Comp Clin Pathol. 2012; 21(6): 1739-1744, doi: 10.1007/ /s00580-011-1359-4.

11. Braga-Neto $M B$, Warren $C A$, Oriá $R B$, et al. Alanylglutamine and glutamine supplementation improves 5-fluorouracil-induced intestinal epithelium damage in vitro. Dig Dis Sci. 2008; 53(10): 2687-2696, doi: 10.1007/ s10620-008-0215-0, indexed in Pubmed: 18320312.

12. Bremer V, Tojo A, Kimura K, et al. Role of nitric oxide in rat nephrotoxic nephritis: comparison between inducible and constitutive nitric oxide synthase. J Am Soc Nephrol. 1997; 8(11): 1712-1721, indexed in Pubmed: 9355074.

13. Cabellos R, Garcia-Carbonero R, Garcia-Lacalle C, et al. Fluorouracil-based chemotherapy in patients with gastrointestinal malignancies: influence of nutritional folate status on toxicity. J Chemother. 2007; 19(6): 744-749, doi: 10.1179/joc.2007.19.6.744, indexed in Pubmed: 18230560.

14. Can C, Sen S, Boztok N, et al. Protective effect of oral $\mathrm{L}$-arginine administration on gentamicin-induced renal failure in rats. Eur J Pharmacol. 2000; 390(3): 327-334, doi: 10.1016/S0014-2999(00)00025-X, indexed in Pubmed: 10708741.

15. Chatterjee PK, Patel NSA, Kvale EO, et al. Inhibition of inducible nitric oxide synthase reduces renal ischemia/ reperfusion injury. Kidney Int. 2002; 61(3): 862-871, doi: 10.1046/j.1523-1755.2002.00234.x, indexed in Pubmed: 11849439.

16. Cherla G, Jaimes EA. Role of L-arginine in the pathogenesis and treatment of renal disease. J Nutr. 2004; 134(10 Suppl): 2801S-2806S; discussion 2818S, indexed in Pubmed: 15465789.

17. Coers W, Timens W, Kempinga C, et al. Specificity of antibodies to nitric oxide synthase isoforms in human, guinea pig, rat, and mouse tissues. J Histochem Cytochem. 1998; 46(12): 1385-1392, doi: 10.1177/002215549804601207, indexed in Pubmed: 9815280.

18. Cool JC, Dyer JL, Xian CJ, et al. Pre-treatment with insulinlike growth factor-I partially ameliorates 5 -fluorouracilinduced intestinal mucositis in rats. Growth Horm IGF Res. 2005; 15(1): 72-82, doi: 10.1016/j.ghir.2004.12.002, indexed in Pubmed: 15701575.

19. Devlin TM. Text book of biochemistry: with clinical correlation, 4th ed. John Wiley and Sons Inc, New York 1997: 553-560.

20. El-Hoseany NMA. Protective effect of captopril against 5-fluorouracil-induced hepato and nephrotoxicity in male Albino rats. J Am Sci. 2012; 8(2): 680-685, doi: 10.7537/j. issn.1545-1003.
21. El-Sayed eS, Abd-Ellah MF, Attia SM. Protective effect of captopril against cisplatin-induced nephrotoxicity in rats. PakJ. Pharm Sci. 2008; 21(3): 255-261, indexed in Pubmed: 18614421.

22. Fujihara CK, Mattar AL, Vieira JM, et al. Evidence for the existence of two distinct functions for the inducible NO synthase in the rat kidney: effect of aminoguanidine in rats with 5/6 ablation. J Am Soc Nephrol. 2002; 13(9): 2278-2287, doi: 10.1097/01.ASN.0000027354.12330. F4, indexed in Pubmed:12191972.

23. Hayashi T, Matsui-Hirai $H$, Fukatsu A, et al. Selective iNOS inhibitor, ONO1714 successfully retards the development of high-cholesterol diet induced atherosclerosis by novel mechanism. Atherosclerosis. 2006; 187(2): 316-324, doi: 10.1016/j.atherosclerosis.2005.10.023, indexed in Pubmed:16325187.

24. Heeringa $\mathrm{P}$, van Goor $\mathrm{H}$, Moshage $\mathrm{H}$, et al. Expression of iNOS, eNOS, and peroxynitrite-modified proteins in experimental anti-myeloperoxidase associated crescentic glomerulonephritis. Kidney Int. 1998; 53(2): 382-393, doi: 10.1046/j.1523-1755.1998.00780.x, indexed in Pubmed: 9461097.

25. Isaka Y, Rakugi H. Severe adverse effects of 5-fluorouracil in S-1 were lessened by haemodialysis due to elimination of the drug. NDT Plus. 2009; 2(2): 152-154, doi: 10.1093/ ndtplus/sfn195, indexed in Pubmed: 25949315.

26. Ito K, Chen J, Vaughan ED, et al. Dietary L-arginine supplementation improves the glomerular filtration rate and renal blood flow after 24 hours of unilateral ureteral obstruction in rats. J Urol. 2004; 171(2 Pt 1): 926-930, doi: 10.1097/01.ju.0000105073.67242.eb, indexed in Pubmed: 14713855.

27. Joles JA, Vos IH, Gröne HJ, et al. Inducible nitric oxide synthase in renal transplantation. Kidney Int. 2002; 61(3): 872-875, doi: 10.1046/j.1523-1755.2002.00235.x, indexed in Pubmed: 11849440.

28. Kakoki M, Kim HS, Arendshorst WJ, et al. L-Arginine uptake affects nitric oxide production and blood flow in the renal medulla. Am J Physiol Regul Integr Comp Physiol. 2004; 287(6): R1478-R1485, doi: 10.1152/ajpregu.00386.2004, indexed in Pubmed: 15319219.

29. Lin $Y$, Wang LN, Xi YH, et al. L-arginine inhibits isoproterenol-induced cardiac hypertrophy through nitric oxide and polyamine pathways. Basic Clin Pharmacol Toxicol. 2008; 103(2): 124-130, doi: 10.1111/j.17427843.2008.00261.x, indexed in Pubmed: 18816294.

30. Ling H, Edelstein C, Gengaro $P$, et al. Attenuation of renal ischemia-reperfusion injury in inducible nitric oxide synthase knockout mice. Am J Physiol. 1999; 277(3 Pt 2): F383-F390, indexed in Pubmed: 10484522.

31. Mansour M, Daba MH, Gado A, et al. Protective effect of L-arginine against nephrotoxicity induced by cyclosporine in normal rats. Pharmacol Res. 2002; 45(6): 441-446, doi: 10.1006/phrs.2002.0968, indexed in Pubmed: 12162943.

32. Miller AA, Megson IL, Gray GA. Inducible nitric oxide synthase-derived superoxide contributes to hypereactivity in small mesenteric arteries from a rat model of chronic heart failure. Br J Pharmacol. 2000; 131(1): 29-36, doi: 10.1038/ sj.bjp.0703528, indexed in Pubmed: 10960065. 
33. Raij L, Baylis C. Glomerular actions of nitric oxide. Kidney Int. 1995; 48(1): 20-32, doi: 10.1038/ki.1995.262, indexed in Pubmed: 7564080

34. Rashid S, Ali N, Nafees S, et al. Mitigation of 5-Fluorouracil induced renal toxicity by chrysin via targeting oxidative stress and apoptosis in wistar rats. Food Chem Toxicol. 2014; 66: 185-193, doi: 10.1016/j.fct.2014.01.026, indexed in Pubmed: 24486618.

35. Romero F, Rodríguez-Iturbe B, Parra G, et al. Mycophenolate mofetil prevents the progressive renal failure induced by $5 / 6$ renal ablation in rats. Kidney Int. 1999; 55(3): 945-955, doi: 10.1046/j.1523-1755.1999.055003945.x, indexed in Pubmed: 10027931.

36. Rusai K, Fekete A, Szebeni B, et al. Effect of inhibition of neuronal nitric oxide synthase and L-arginine supplementation on renal ischaemia-reperfusion injury and the renal nitric oxide system. Clin Exp Pharmacol Physiol. 2008; 35(10): 1183-1189, doi: 10.1111/j.14401681.2008.04976.x, indexed in Pubmed: 18518882.

37. Saleh S, El-Demerdash E. Protective effects of L-arginine against cisplatin-induced renal oxidative stress and toxicity: role of nitric oxide. Basic Clin Pharmacol Toxicol. 2005; 97(2): 91-97, doi: 10.1111/j.1742-7843.2005.pto_114.x, indexed in Pubmed: 15998355.

38. Schneider R, Raff $U$, Vornberger $N$, et al. L-Arginine counteracts nitric oxide deficiency and improves the recovery phase of ischemic acute renal failure in rats. Kidney Int. 2003; 64(1): 216-225, doi: 10.1046/j.1523-1755.2003.00063.x, indexed in Pubmed: 12787412.

39. Schramm L, La M, Heidbreder E, et al. L-arginine deficiency and supplementation in experimental acute renal failure and in human kidney transplantation. Kidney
Int. 2002; 61(4): 1423-1432, doi: 10.1046/j.1523-1755.2002.00268.x, indexed in Pubmed: 11918749.

40. Schwartz D, Mendonca M, Schwartz I, et al. Inhibition of constitutive nitric oxide synthase (NOS) by nitric oxide generated by inducible NOS after lipopolysaccharide administration provokes renal dysfunction in rats. J Clin Invest. 1997; 100(2): 439-448, doi: 10.1172/JCI119551, indexed in Pubmed:9218522.

41. Sharma SP. Nitric oxide and the kidney. Indian J Nephrol. 2004; 14(3): 77-84.

42. Silverstein RA, González de Valdivia E, Visa N. The incorporation of 5-fluorouracil into RNA affects the ribonucleolytic activity of the exosome subunit Rrp6. Mol Cancer Res. 2011; 9(3): 332-340, doi: 10.1158/1541-7786.MCR-100084, indexed in Pubmed: 21289297.

43. Thant AA, Wu Y, Lee J, et al. Role of caspases in 5-FU and selenium-induced growth inhibition of colorectal cancer cells. Anticancer Res. 2008; 28: 3579-3592, indexed in Pubmed: 3771536.

44. Vaziri ND, Ni Z, Wang XQ, et al. Down-regulation of nitric oxide synthase in chronic renal insufficiency: role of excess PTH. Am J Physiol. 1998; 274(4 Pt 2): F642-F649, indexed in Pubmed: 9575886.

45. Xia $Y$, Roman $\sqcup$, Masters BS, et al. Inducible nitric-oxide synthase generates superoxide from the reductase domain. J Biol Chem. 1998; 273(35): 22635-22639, doi: 10.1074/ jbc.273.35.22635, indexed in Pubmed: 9712892.

46. Xian CJ, Howarth GS, Cool JC, et al. Effects of acute 5-fluorouracil chemotherapy and insulin-like growth factor-I pretreatment on growth plate cartilage and metaphyseal bone in rats. Bone. 2004; 35(3): 739-749, doi: 10.1016/j. bone.2004.04.027, indexed in Pubmed: 15336611. 